\title{
Decoupling Gas Evolution from Water-Splitting Electrodes
}

Pablo Peñas, Peter van der Linde, Wouter Vijselaar, Devaraj van der Meer, Detlef Lohse, Jurriaan Huskens, Han Gardeniers, Miguel Modestino, David Fernandez Rivas

Submitted date: 13/08/2019 - Posted date: 14/08/2019

Licence: CC BY-NC-ND 4.0

Citation information: Peñas, Pablo; Linde, Peter van der; Vijselaar, Wouter; van der Meer, Devaraj; Lohse, Detlef; Huskens, Jurriaan; et al. (2019): Decoupling Gas Evolution from Water-Splitting Electrodes. ChemRxiv. Preprint.

Bubbles are known to hinder electrochemical processes in water-splitting electrodes. In this study, we present a novel method to promote gas evolution away from the electrode surface. We consider a ring microelectrode encircling a hydrophobic microcavity from which a succession of bubbles grows. The ring microelectrode, tested under alkaline water electrolysis conditions, does not suffer from bubble coverage. Consequently, the chronopotentiometric fluctuations of the cell are weaker than those associated with conventional microelectrodes. Herein, we provide fundamental understanding of the mass transfer processes governing the transient behaviour of the cell potential. With the help of numerical transport models, we demonstrate that bubbles forming at the cavity reduce the concentration overpotential by lowering the surrounding concentration of dissolved gas, but may also aggravate the ohmic overpotential by blocking ion-conduction pathways. The theoretical and experimental insight gained have relevant implications in the design of efficient gas-evolving electrodes.

File list (2)

RingElectrodesVSub.pdf (2.04 MiB) view on ChemRxiv • download file 


\title{
Decoupling Gas Evolution from Water-Splitting Electrodes
}

\author{
Pablo Peñas $\dagger^{\mathrm{a}}$, Peter van der Linde $\dagger^{\mathrm{b}}$, Wouter Vijselaar ${ }^{\mathrm{d}}$, Devaraj van der Meer $^{\mathrm{a}}$, Detlef Lohse ${ }^{\mathrm{a}}$, Jurriaan \\ Huskens $^{\mathrm{d}}$, Han Gardeniers ${ }^{\mathrm{b}}$, Miguel A. Modestino ${ }^{\mathrm{c}}$, and David Fernández Rivas ${ }^{\mathrm{b}}$ \\ ${ }^{a}$ Physics of Fluids Group and Max Planck Center Twente, MESA+ Institute and J. M. Burgers Centre for Fluid \\ Dynamics, Faculty of Science and Technology, University of Twente, P.O. Box 217, 7500 AE Enschede, The Netherlands \\ ${ }^{\mathrm{b}}$ Mesoscale Chemical Systems, MESA + Institute, University of Twente, Enschede, The Netherlands \\ ${ }^{\mathrm{c}}$ New York University, Tandon School of Engineering, 6 MetroTech Center, Brooklyn, NY 11201, United States of America \\ ${ }^{\mathrm{d}}$ Molecular Nanofabrication, University of Twente, Enschede, The Netherlands \\ ${ }^{\dagger}$ These authors contributed equally to this work.
}

August 13, 2019

\begin{abstract}
Bubbles are known to hinder electrochemical processes in water-splitting electrodes. In this study, we present a novel method to promote gas evolution away from the electrode surface. We consider a ring microelectrode encircling a hydrophobic microcavity from which a succession of bubbles grows. The ring microelectrode, tested under alkaline water electrolysis conditions, does not suffer from bubble coverage. Consequently, the chronopotentiometric fluctuations of the cell are weaker than those associated with conventional microelectrodes. Herein, we provide fundamental understanding of the mass transfer processes governing the transient behaviour of the cell potential. With the help of numerical transport models, we demonstrate that bubbles forming at the cavity reduce the concentration overpotential by lowering the surrounding concentration of dissolved gas, but may also aggravate the ohmic overpotential by blocking ion-conduction pathways. The theoretical and experimental insight gained have relevant implications in the design of efficient gas-evolving electrodes.
\end{abstract}

\section{Introduction}

The uncontrolled formation of bubbles on catalyst surfaces can take a remarkable toll on the efficiency of many gas-evolving electrochemical systems [1] among which photoelectrochemical cells stand out in significance [2]. These surface bubbles not only reduce the electrochemically active contact area between the electrode and the reacting liquid, but also block ion-conduction pathways, thereby increasing the ohmic drop across the electrolyte in the cell [3]. In some cases, bubble formation can even cause direct catalyst degradation [4].

Despite the extensive research done on electrolytic bubbles, the bubble problem is far from being solved $[4,5]$. One approach is to micropattern the electrode with hydrophobic sites to promote bubble growth at desired locations [6]. The effectiveness of microcavities etched on silicon substrates, first employed as a means to control multibubble surface cavitation [7], has been recently tested under electrolysis conditions [8]. A second approach is the implementation of superwetting electrodes [1] in order to minimise the fraction of bubble coverage.

In contrast, here we choose to mitigate the effect of bubble formation in water-splitting electrodes by promoting the nucleation of bubbles away from the electrode surface. This was achieved by means of a ring microelectrode encircling a superhydrophobic microcavity etched on a hydrophilic silicon substrate. During electrolysis, a sequence of bubbles forms on the cavity and not elsewhere, precisely because the energy landscape for nucleation is most favourable there. The electrode surface therefore remains unspoiled. These bubbles play a crucial role in the electrolysis process by actively lowering the concentration of dissolved gas around them as they grow. The likeliness of another bubble nucleating on the ring electrode is thereby substantially diminished. In this work we will provide insights into the relationship between the response of the electrochemical cell to the various mass transfer processes surrounding the ring-cavity configuration under constant-current electrolysis.

The main advantage of such a configuration is that the ring electrode does not suffer from any ohmic penalties associated with bubble coverage [9], nor from the large fluctuations in the surface overpotential that usually coexist with them. For instance, bubbles detaching from microelectrodes have been reported to induce prominent positive current peaks under potentiostatic conditions [10,11], or negative peaks in the overpotential under galvanostatic conditions [12]. In these cases, much of the cyclic variation of the surface overpotential is by virtue of the high bubble coverage fraction, or rather by the reduction and subsequent liberation of a substantial portion of the electrode active area as a bubble grows and detaches. 


\section{Experimental}

\section{Ring microelectrode microfabrication}

The electrode consists of a thin platinum ring (inner radius $r_{i}=400 \mu \mathrm{m}$, outer radius $r_{e}=410 \mu \mathrm{m}$, surface area $A=0.0254 \mathrm{~mm}^{2}$ ) encircling a hydrophobic cavity or pit (radius $r_{p}=15 \mu \mathrm{m}$ ) etched on a flat and hydrophilic $10 \times 10 \mathrm{~mm}^{2} \mathrm{SiO}_{2}$ substrate. A lead connects the ring to an insulated electrical contact pad $\left(2 \times 2 \mathrm{~mm}^{2}\right)$ located in a corner of the substrate surface. Details of its microfabrication can be found in appendix A.

\section{Alkaline electrolysis cell}

The substrate was placed at the bottom of an alkaline water electrolysis cell, sketched in Figure 1 . The cell is enclosed by an open $20 \times 60 \times 20 \mathrm{~mm}^{3}$ glass container. The ring electrode acts as the hydrogen-evolving cathode; a platinum wire embodies the oxygen-evolving anode. The anode has a large surface area $\left(\sim 8 \mathrm{~mm}^{2}\right)$, specifically over 300 times greater than that of the ring cathode. Both electrodes are connected through a Keithley 2410 power source, and kept at a distance $\sim 1 \mathrm{~cm}$ apart. At the beginning of each experiment, $15 \mathrm{~mL}$ of fresh electrolyte was poured into the cell, resulting in a $\sim 12.5 \mathrm{~mm}$ layer of electrolyte above the substrate. The electrolyte was prepared by dissolving $0.01 \mathrm{M} \mathrm{NaHCO}_{3}$ in Milli-Q purified water.

Sodium bicarbonate completely dissociates into sodium $\left(\mathrm{Na}^{+}\right)$and bicarbonate $\left(\mathrm{HCO}_{3}{ }^{-}\right)$ions, which constitute the vast majority of the supporting ions. The electrolyte is slightly basic in nature, with a measured $\mathrm{pH}$ 7.4 , due to the equilibrium between $\mathrm{OH}^{-}, \mathrm{HCO}_{3}{ }^{-}$and $\mathrm{CO}_{3}{ }^{2-}$ ions in solution. The equilibrium reactions are detailed in the Supporting Information.

In each of the three experiments reported in this paper, the cell was operated at a constant current, namely, at $I=5,10$ or $20 \mu \mathrm{A}$. The corresponding current densities at the ring electrode are 19.6, 39.3 and $78.6 \mathrm{~mA} / \mathrm{cm}^{2}$ respectively. All three current densities were found to be sufficiently low as to prevent bubbles from nucleating on the surface of both the ring cathode and the anode. However, these current densities did allow for a single bubble from spontaneously growing from the hydrophobic pit a few seconds after the start of electrolysis, as evidenced in Figure 2. This is a clear indication that the surrounding electrolyte is henceforth sufficiently saturated with dissolved hydrogen as to sustain bubble growth. Eventually, the bubble detaches once it reaches the critical size at which the buoyancy force exceeds the maximal interfacial tension force that the triple contact line at the rim of the pit is able to provide [13]. Shortly afterwards, a new bubble nucleates and the process repeats itself over again. The cell voltage and the growth of first three bubbles of the succession were recorded simultaneously. These are shown in Figure 3.

The evolution of $\mathrm{H}_{2}$ gas abides by the reversible half reaction occurring at the cathode of the alkaline electrolysis cell $[14,15,16]$,

$$
\mathrm{H}_{2} \mathrm{O}+2 \mathrm{e}^{-} \rightleftharpoons 2 \mathrm{OH}^{-}+\mathrm{H}_{2}
$$

The hydroxyl ion, $\mathrm{OH}^{-}$, is thus produced and consumed at the cathode and anode respectively (cf. Figure 1), at equal rates. In choosing galvanostatic electrolysis, the production rate of $\mathrm{H}_{2}$ gas remains constant in time. In the absence of convection, but in the presence of an electric field, the current flowing through a cathode of surface area $A$ is related to the surface concentration and gradient of the (produced) species according to

$$
\frac{I / A}{n_{j} F}=J_{j}=-\frac{D_{j}}{A} \int_{\Sigma}\left(\frac{\partial c_{j}}{\partial x}+\frac{z_{j} F}{R T} c_{j} \frac{\partial \phi}{\partial x}\right) \mathrm{d} \sigma, \quad j=\mathrm{H}_{2}, \mathrm{OH}^{-}
$$

where $J_{j}$ denotes the average molar flux of species $j, F=96485 \mathrm{C} / \mathrm{mol}$ is Faraday's constant and $n_{\mathrm{H}_{2}}=2$, $n_{\mathrm{OH}^{-}}=1$ are stoichiometric constants. The second equality constitutes the Nernst-Planck flux equation [17], where $c_{j}$ is the concentration, $\Sigma$ describes the cathode surface, $x$ is the coordinate pointing normally outwards from $\Sigma$, and $\mathrm{d} \sigma$ is an infinitesimal area element. The electric potential is denoted by $\phi$ and $z_{j}$ is the charge of the species: $z_{\mathrm{H}_{2}}=0, z_{\mathrm{OH}^{-}}=-1 ; R=8.314 \mathrm{Jmol}^{-1} \mathrm{~K}^{-1}$ is the gas constant and $T$ the absolute temperature. The transport of uncharged species $\left(z_{j}=0\right)$ such as $\mathrm{H}_{2}$ is strictly driven by diffusion: the migration flux component is identically zero. For the case of $\mathrm{OH}^{-}$, Equation (2) is only applicable for large current densities at which the large local overconcentration of $\mathrm{OH}^{-}$overwhelms the buffer capacity of the surrounding electrolyte. Otherwise, most of the excess $\mathrm{OH}^{-}$immediately recombines into $\mathrm{HCO}_{3}{ }^{-}$and $\mathrm{CO}_{3}{ }^{2-}$ ions to satisfy chemical equilibria (see Supporting Information).

\section{Results and discussion}

\section{Bubble growth dynamics}

We begin with a treatment of the efficiency of gas evolution [18] and the bubble growth dynamics observed in our experiments (see Figure 3). The number of moles of $\mathrm{H}_{2}$ gas, $N_{d}$, in a spherical bubble at its detachment radius $a_{d}$ can be computed from the ideal gas law,

$$
\frac{4}{3} \pi a_{d}^{3} p=N_{d} R T
$$

where $p \simeq 1$ bar is the bubble pressure and $T=293 \mathrm{~K}$. In truth, the assumption that there are no other gases present in the bubble is violated especially in the case of the first bubble since the electrolyte is equilibrated with air. The presence of dissolved air explains why the first hydrogen bubble nucleates and grows just a few 
seconds after the start of electrolysis [8]. After this start-up effect, the mean efficiency of bubble evolution can be computed as the amount of $\mathrm{H}_{2}$ in the bubble at the time of detachment divided by the total amount of gas evolved during the bubble lifetime $t_{d}$ :

$$
\eta=\frac{N_{d}}{A J_{\mathrm{H}_{2}} t_{d}}=\frac{4 \pi}{3} \frac{a_{d}^{3} p}{R T} \frac{n_{\mathrm{H}_{2}} F}{I t_{d}} .
$$

For a typical bubble, $a_{d} \sim 0.5 \mathrm{~mm}, t_{d} \sim 1000-3000 \mathrm{~s}$. We then obtain, for the experimental current densities, $\eta \approx 30 \%$, which implies that roughly $30 \%$ of the evolved hydrogen eventually ends up in the bubble, while the rest is being continuously absorbed by the bulk fluid. The bubble hence mainly grows by diffusion, absorbing gas from the bulk. The efficiency of our configuration is still not close to the $100 \%$ efficiency that is practically observed in conventional microelectrodes upon which (single) bubbles form $[12,11]$. Nonetheless, it stands an order of magnitude higher than in the case of a single bubble nucleating on a flat planar electrode much larger than the bubble itself [19].

We can go further and compare the bubble growth dynamics with the Epstein-Plesset theory [20]. For slow growth dynamics, the bubble radius of the $n$th bubble in the succession can be assumed to grow in time as $[21,8]$

$$
a_{n}(t) \approx \sqrt{2 \Lambda \zeta_{n} D_{\mathrm{H}_{2}} t_{n}}
$$

from which one may estimate the effective $\mathrm{H}_{2}$ gas supersaturation of the bulk surrounding the $n$th bubble in the succession, that is,

$$
\zeta_{n}=\bar{C}_{n} / k_{\mathrm{H}_{2}} p-1 .
$$

Here, $\bar{C}_{n}(t)$ represents the effective far-field concentration of dissolved $\mathrm{H}_{2}$ gas surrounding the bubble, $k_{\mathrm{H}_{2}}$ denotes Henry's coefficient of $\mathrm{H}_{2}$ in water, $\Lambda=k_{\mathrm{H}_{2}} R T=0.0188$ is the Ostwald coefficient and $t_{n}$ is the time elapsed since nucleation. The theoretical fits of (5) to the experimental bubble growth rates yields a characteristic supersaturation value of $\zeta \sim 0.44$ (first bubble) and 0.54 (third bubble) for $19.6 \mathrm{~mA} / \mathrm{cm}^{2}$. For 39.3 and $78.6 \mathrm{~mA} / \mathrm{cm}^{2}$, we obtain $\zeta \sim 0.62-0.71$ and $1.77-1.89$, respectively. The higher growth rates of the subsequent bubbles in the succession is a clear indicator that the bulk hydrogen concentration near the ring electrode is indeed increasing in time.

\section{Cell potential}

Figure 3 reveals a transient 'diffusion-like' behaviour of the cell voltage. Remarkably, a steady-state value is never reached during the long time scale of our experiment. The cell voltage is clearly influenced by the presence of bubbles, not only by bubble growth, as one may infer by the periodic relaxation of the potential in the timescale of the bubbles' lifetime, but also by bubble detachment, which is clearly synchronous with sudden drops in the voltage.

In order to address these matters further, we must first acknowledge the different contributions to the cell potential [14]:

$$
E(t)=E_{\Omega}(t)+E_{a}(t)-E_{c}(t) .
$$

The anode and cathode potential are denoted by $E_{a}$ and $E_{c}$ respectively; $E_{\Omega}(t)$ refers to the ohmic overpotential. The dependency of the electrode potential on the current density, surface concentrations and reaction rates can be modelled by the most general form of the Butler-Volmer equation [17]. The electrode kinetic properties of our electrodes remain unknown, and it is not the purpose of this paper to determine them. Nonetheless, it stands to reason that the influence of bubble growth or detachment on the cell potential can be made manifest through the concentration overpotential, only.

Therefore, as a first approximation, we can assume that at a given current density the reaction overpotential of the ring cathode remains fairly constant. The concentration overpotential, however, can be assumed to behave semi-quantitatively in the same way to the Nernst (equilibrium) potential [16],

$$
E_{c}(t)=E_{0}+\frac{R T}{2 F} \ln \left(\frac{\left(C_{\mathrm{H}_{2} \mathrm{O}}\right)^{2}}{\left(C_{\mathrm{OH}^{-}}(t)\right)^{2} C_{\mathrm{H}_{2}}(t)}\right)
$$

where $C_{j}$ denotes the concentration of species $j$ at the cathode surface and $E_{0}$ is the (unknown) standard potential of our cathode. It is worth pointing out that platinum electrodes driving water electrolysis in acidic conditions have extremely fast reaction kinetics and the Butler-Volmer equation in fact reduces to the Nernst equation. In alkaline media, however, reaction kinetics are several orders of magnitude slower [22], and the reaction overpotentials are non-negligible. In any case, (8) conveys the important fact that an increase in the surface concentrations of $\mathrm{H}_{2}$ or $\mathrm{OH}^{-}$renders an increase of the cell voltage $E$. The concentration of water in the electrolyte is naturally so high that it can be assumed constant and uniform, $C_{\mathrm{H}_{2} \mathrm{O}} \simeq 55.5 \mathrm{M}$.

The Nernst potential at the anode is given by a similar equation. We may however neglect the concentration overpotential on the anode potential caused by the unsteady surface concentrations of $\mathrm{O}_{2}$ and $\mathrm{OH}^{-}$. This is justified by noting that changes in the surface concentration of the reacting species are proportional to the current density of the electrode (see Equation 12). In our case, $I / A$ is a factor of 300 smaller at the anode.

By predicting then the evolution of $C_{\mathrm{H}_{2}}(t)$ and any changes in the ionic concentration distribution, it is possible to estimate, semi-quantitatively, the corresponding variation in the concentration and ohmic overpotential 
in time. The evolution of the concentration $c_{j}$ of each (ionic) species is governed by the advection-diffusionmigration equation, commonly referred to as the Nernst-Planck equation,

$$
\frac{\partial c_{j}}{\partial t}=D_{j} \nabla^{2} c_{j}+\nabla \cdot\left(\frac{D_{j} z_{j} F}{R T} c_{j} \nabla \phi\right)-\boldsymbol{U} \cdot \nabla c_{j}+S_{j}
$$

along with the electroneutrality condition, $\sum_{j} z_{j} c_{j}=0[23]$. The velocity field, if any flow is present, is denoted by $\boldsymbol{U}$. Any equilibrium reactions between the different ions are taken into account through the source term $S_{j}$ (see Supporting Information).

We advance that the transient behaviour of the potential observed in Figure 3 can be explained by three main factors. The first is the concentration overpotential on the cathode, as a consequence of the diffusiondriven evolution of surface concentration of $\mathrm{H}_{2}$ and, to a lesser extent, $\mathrm{OH}^{-}$. The concentration overpotential is mitigated by the presence of the bubbles growing on the cavity which essentially act as a sink of hydrogen gas. The second factor is the migration-driven transport of the supporting ions from the anode to the cathode across the bulk of the electrolyte, consequently increasing the ohmic overpotential over time. Last but not least is the advection induced by a detaching bubble [24]. Its repercussion is twofold: it not only disrupts the high-concentration $\mathrm{H}_{2}$ diffusion layer at the electrode, but also alleviates the high ionic concentration layer that inevitably surrounds the electrode. These arguments are developed and justified next.

\section{The concentration overpotential in the presence of bubbles}

We commence estimating the relaxation time for the concentrations of $\mathrm{H}_{2}$ on the ring electrode surface to reach the steady value in the hypothetical absence of bubbles. The transport equation (9) for $j=\mathrm{H}_{2}$ simplifies to the diffusion equation

$$
\frac{\partial c_{\mathrm{H}_{2}}}{\partial t}=D_{\mathrm{H}_{2}} \nabla^{2} c_{\mathrm{H}_{2}},
$$

for which $D_{\mathrm{H}_{2}} \sim 5.0 \times 10^{-9} \mathrm{~m}^{2} / \mathrm{s}$ [25]. We can immediately resort to the analytical solution of Equation (10) concerning an equivalent hemispherical electrode of area $A=2 \pi r_{e}^{2}$ in a semi-infinite medium. The electrode is producing $\mathrm{H}_{2}$ at a constant flux $I / A n_{\mathrm{H}_{2}} F$ and the initial bulk concentration is $C_{\mathrm{H}_{2}}^{*}$. It can be shown, by the method of Laplace transforms, that the surface concentration $C_{\mathrm{H}_{2}}$ evolves in time as

$$
\frac{C_{\mathrm{H}_{2}}(t)-C_{\mathrm{H}_{2}}^{*}}{C_{\mathrm{H}_{2}}^{s s}-C_{\mathrm{H}_{2}}^{*}}=1-\exp \left(\frac{D_{\mathrm{H}_{2}} t}{r_{e}^{2}}\right) \operatorname{erfc}\left(\frac{\sqrt{D_{\mathrm{H}_{2}} t}}{r_{e}}\right)
$$

where $C_{\mathrm{H}_{2}}^{s s}$ is the steady-state surface concentration. Equation (11) is plotted in Figure 4 and compared with the surface concentrations expected for a thin ring $\left(r_{i} / r_{e}=0.976\right)$ and a disk $\left(r_{i} / r_{e}=0\right)$ electrode. These were computed numerically using the open-source partial differential equation solver FreeFem $++[26]$. Recall that $r_{e}$ refers to the outer radius of the ring or disk.

From Figure 4 we estimate that the dimensionless relaxation time of the surface concentration is $D_{\mathrm{H}_{2}} t / r_{e}^{2} \sim$ 50. One would then expect the concentration profile and concentration overpotential to reach the steady state in about $t \sim 30$ minutes in the absence of bubbles. Our experiments in Figure 3 show otherwise: the overall cell potential and bubble growth rates enduringly increase during a much greater time scale. This suggests that the bubbles impede the surface concentration from attaining a steady-state value so quickly.

The prompt increase of the cell voltage observed immediately after the start of electrolysis (cf. Figure 3) can be mainly attributed to the concentration overpotential. This is justified by the very rapid initial diffusiondriven increase of $C_{\mathrm{H}_{2}}$ as seen in Figure 4. Soon after, however, a bubble nucleates and grows at the centre of the ring. The bubble essentially acts as a sink of $\mathrm{H}_{2}$ gas which has a depressing effect on $C_{\mathrm{H}_{2}}$ and hence on the concentration overpotential. Indeed, in this configuration where the bubble does not mask the electrode, the bubble can only be beneficial for the potential and the efficiency of the system as it passively removes $\mathrm{H}_{2}$ from the vicinity of electrode surface. This is precisely the 'enhancement effect' referred to by other authors $[27,10]$.

The enhancement effect is perhaps even better conveyed by Figure 5 which compares the $\mathrm{H}_{2}$ concentration field in the vicinity of the ring electrode in the absence and in the presence of a bubble close to detaching. The bubble was treated as a stationary boundary under the pseudosteady-state approximation [27]. This is justified as long as the bubble lifetime $t_{d}$ notably exceeds the relaxation time by diffusion of the concentration field around a bubble, $\tau_{a}=a_{d}^{2} / D_{\mathrm{H}_{2}}$. In our case, we find that $t_{d} / \tau_{a}>10$. The (axisymmetric) diffusion equation 10 could then be readily solved in FreeFem++, on a $50 r_{e}$ square domain, subject to a constant flux condition at the electrode surface, and imposing a constant saturation concentration of $k_{\mathrm{H}_{2}} p=0.77 \mathrm{mM}$ at the bubble surface (see Supporting Information for details).

The simulation snapshots are taken at a dimensionless time $D_{\mathrm{H}_{2}} t / r_{e}^{2}=100$, i.e., approximately one hour in dimensional time, which is roughly the lifetime of our bubbles at the lowest current density. This implies that the concentration field in the absence of the bubble (figure $5 a$ ) is essentially the steady-state solution.

Figure 5(b) highlights the enormous influence that the bubble has on the concentration field. Strikingly, the bubble lowers the electrode surface concentration to almost half of the bubble-free steady-state value. In fact, the steady-state surface concentration of an electrode in a semi-infinite medium initially at $C_{\mathrm{H}_{2}}^{*}$ is given by the analytical expression

$$
\frac{I / A}{n_{\mathrm{H}_{2}} F}=\beta \frac{D_{\mathrm{H}_{2}}}{r_{e}}\left(C_{\mathrm{H}_{2}}^{s s}-C_{\mathrm{H}_{2}}^{*}\right),
$$

where the geometric factor $\beta$ accounts for the electrode geometry. For a hemispheric electrode, $\beta=1$ [17], a circular disk electrode has $\beta=4 / \pi$ [28], whereas our ring electrode of thickness $r_{i} / r_{e}=0.976$ has $\beta \simeq 18.3$ 
(see appendix B). In the hypothetical absence of bubbles, Equation (12) with $C_{\mathrm{H}_{2}}^{*}=0$ yields $C_{\mathrm{H}_{2}}^{s s} \approx 4.6$, 9.2 and $18.3 \mathrm{mM}$ for $I / A=19.7,39.3$ and $78.6 \mathrm{~mA} / \mathrm{cm}^{2}$ respectively. These concentrations correspond to steady-state supersaturations of $\zeta=C_{\mathrm{H}_{2}}^{s s} / k_{\mathrm{H}_{2}} p-1 \sim 5,11$, and 23. Notice that even when halving these values (to account for the presence of bubbles), the electrode surface supersaturations remain much larger than the effective supersaturations surrounding the first bubbles in the succession (approximately 0.5, 0.7 and 1.8 respectively) previously calculated (cf. subsection "Bubble growth dynamics").

The origin of this mismatch lies in that the highest concentrations and concentration gradients are contained within a small diffusion boundary layer surrounding the ring electrode. Figure $5(b)$ reveals that this diffusion layer is quite small in comparison to the bubble size at detachment. The size of the diffusion layer thickness is often characterized by the Nernst diffusion layer thickness $\delta[29,17,24]$. We find that the Nernst diffusion thickness, which adopts the formal definition of $\delta / r_{e}=\beta \sim 0.05$ in compliance with (12), indeed represents a realistic length scale of the diffusion layer observed in our thin-ring configuration.

We finally turn our attention to the sudden drops in the cell voltage caused by bubble detachment, or rather by the forced convection that is generated during the departure process. As discussed in the introduction, the reduction in the surface overpotential is less prominent than in the conventional case of a bubble departing from a microelectrode $[12,11]$, owing to the fact that the ring electrode is never in direct contact with the bubble.

Even without considering entrainment in the bubble wake, whose velocities at a fixed height near the electrode were incidentally measured to decay exponentially over a couple of seconds (data not shown), we expect the $\mathrm{H}_{2}$ diffusion layer to be completely disrupted by the detaching bubble. The volume occupied by the detaching bubble must be refilled with the adjacent electrolyte, including the electrolyte in the diffusion layer around the electrode due to its immediate proximity. We find justification in the fact that the volume of the half-torus (with tube radius $\delta$ ) comprising the Nernst diffusion layer surrounding the electrode is very small compared to the bubble volume at detachment:

$$
\frac{V_{N}}{V_{d}} \sim \frac{\pi^{2} r_{e} \delta^{2}}{4 \pi a_{d}^{3} / 3}=\frac{3 \pi}{4 \beta^{2}} \frac{r_{e}^{2}}{a^{3}} \sim 2 \times 10^{-3} .
$$

\section{Ohmic overpotential}

It is expected that detachment-driven convection additionally disrupts the diffusion layer of high ionic concentration that surrounds the cathode, thereby reducing the ohmic overpotential, $E_{\Omega}$ in Equation (7). This claim is evidenced in Figure 6, which compares the steady-state concentration field of cations, or equivalently anions, in the presence and absence of a bubble. The concentration field is obtained from a simple numerical model. The bubble is treated as a stationary boundary as before, but now the medium is confined to a closed cylindrical domain that resembles our experimental configuration. In particular, the interelectrode distance, the volume of solution, and electrode sizes are equivalent to those of our set-up. The equilibrium concentrations of $\mathrm{Na}^{+}$ and $\mathrm{HCO}_{3}{ }^{-}$ions are assumed to always remain much greater than those of $\mathrm{OH}^{-}, \mathrm{CO}_{3}{ }^{2-}$ and $\mathrm{H}^{+}$. Upon the approximation that the solution has a perfect buffer capacity, it can be shown (see Supporting Information) that the steady-state Nernst-Planck equation (9) simplifies to

$$
\nabla^{2} c_{ \pm}=0
$$

where $c_{ \pm} \simeq c_{\mathrm{Na}^{+}} \simeq c_{\mathrm{HCO}_{3}}$ - is the total cationic or anionic concentration. Equation (14) is solved numerically assuming uniform ionic fluxes at the electrodes (cf. Supporting Information). The aforementioned assumption of perfect buffer capacity is expected to hold everywhere except in the very small diffusion layer closest to the ring cathode surface where the local concentrations of $\mathrm{OH}^{-}$and $\mathrm{CO}_{3}{ }^{2-}$ are highest, even possibly exceeding the concentration of $\mathrm{HCO}_{3}{ }^{-}$for the larger current densities. This is corroborated by Figure 6 , where it is seen that most of the interelectrode concentration difference takes place within this small region. In fact, the dimensionless difference of $\Delta \widetilde{c} \approx 0.07$ obtained for our geometry translates to a fractional concentration difference of order unity since, for our experimental current densities,

$$
\frac{\Delta C_{ \pm}^{*}}{C_{ \pm}^{*}}=\frac{(I / A) r_{e} \Delta \widetilde{c}_{ \pm}}{2 F D_{-} C_{ \pm}^{*}} \sim 1
$$

We have set $C_{ \pm}^{*}=0.01 \mathrm{M}$ as the initial bulk concentration of cations/anions in the solution, whereas $D_{-}$ represents the effective diffusion coefficient that determines the ionic boundary layer thickness at the electrodes ( $D_{-} \simeq D_{\mathrm{HCO}_{3}-}$ under the assumption of perfect buffer). A fractional concentration change of order unity suggests that the local $\mathrm{OH}^{-}$overconcentration near the cathode is likely too large for the buffer capacity of the solution (cf. Supporting Information). In such a case, the validity of perfect buffer assumption of the model is therefore somewhat limited. Nonetheless, this highlights the importance of $C_{ \pm}^{*}$ being sufficiently in excess to ensure weak ionic gradients and a high buffer capacity throughout the whole solution.

Comparing Figures 6(b) and 6(c) one finds that the ionic concentration difference between the cathode ring and anode wire is mildly aggravated when the bubble is present. The bubble seems to block ion-conduction pathways; the high ionic concentration region near the ring visibly increases as a result. Bubble departure will therefore weaken the concentration difference between the cathode and anode, and hence the ohmic drop across the solution. The latter can be readily estimated by solving the steady-state electric potential in the same domain, under the same aforementioned assumptions. It then follows that $\boldsymbol{\nabla} \cdot\left(c_{ \pm} \boldsymbol{\nabla} \phi\right)=0$, from which $\phi$ can be computed given that $c_{ \pm}$is already known. As before, we make the approximation of uniform current density across the electrodes' surface, as opposed to constant potential. 
The steady-state potential difference $\Delta \phi / R T$ can only depend on the geometry of the cell and, very importantly, on the level of electrolyte concentration [30]:

$$
\Gamma=\frac{F D_{-} C_{ \pm}^{*}}{(I / A) L}
$$

where $L$ defines a characteristic length, e.g. the interelectrode distance. We find that $\Delta \phi / R T$ depends inversely on $\Gamma$ : a higher value of $\Gamma$ means that the bulk concentration of supporting ions is more in excess relative to the current density employed. Consequently, both the obstruction effect on the overpotential and the ohmic overpotential itself decrease. Note that $\Gamma$ scales precisely as the inverse of the fractional concentration change defined in (15), a quantity which ideally must be kept small to ensure $E_{\Omega}$ remains small. A value of $\Gamma=0.002$ was used to model our experiments; Figure 7 represents the steady-state electric potential field obtained by numerically solving the steady-state Nernst-Planck equation (9) as explained in the Supplementary Information. At this low value of $\Gamma$, the obstruction due to the mere presence of the bubble can have a remarkable impact on the ohmic overpotential. Indeed, the bubble behaves as an electrical shield, causing the ohmic potential to increase by roughly $20 \%$. Moreover, taking $\Delta \widetilde{\phi} \sim 25$, we expect the magnitude of the ohmic overpotential in our experiments to be of order $E_{\Omega} \sim F \Delta \widetilde{\phi} / R T \sim 0.6 \mathrm{~V}$. Doubling the value of $\Gamma=0.002$, we obtain a reduction of $75 \%$ in the potential difference. This hints that the concentration of supporting electrolyte used in all three experiments $(0.01 \mathrm{M})$ falls short from the optimal level of excess.

Finally, we determine whether the transient nature of the measured cell voltage can be attributed to the unsteadiness in the ohmic potential. In other words, we seek the characteristic time scale required for the potential and ionic concentrations to reach the steady state. Initially, the supporting ions are homogeneously distributed in the solution, compliant with diffusive equilibrium. During electrolysis, however, the diffusion of supporting ions becomes a reactive transport mechanism that opposes their migration due to the presence of the electric field. The steady-state concentration and potential fields are precisely attained once the concentration gradients are large enough for the diffusion fluxes to match and oppose the migratory fluxes of all ionic species everywhere in the solution. In short, migration is the driving transport mechanism of the supporting ions during electrolysis. Therefore, one should only consider the unsteady and migration terms in the Nernst-Plank equation (9) when seeking the ionic relaxation time. These two terms scale as

$$
\frac{\partial c_{ \pm}}{\partial t} \sim \frac{\Delta C_{ \pm}^{*}}{\tau_{M}}, \quad \nabla \cdot\left(\frac{D_{ \pm} z_{ \pm} F}{R T} c_{ \pm} \nabla \phi\right) \sim \frac{D_{ \pm}\left|z_{ \pm}\right| F}{R T} C_{ \pm}^{*} \frac{E_{\Omega}}{L^{2}} .
$$

The characteristic length of the concentration and potential gradients can be taken as $L \sim 1 \mathrm{~cm}$, namely, the distance between the electrodes, whereas $\Delta C_{ \pm}^{*}$ represents the characteristic concentration change that takes place over the migration time scale $\tau_{M}$. An order of magnitude balance between the unsteady and migration terms yields

$$
\tau_{M} \sim\left(\frac{\Delta C_{ \pm}^{*}}{C_{ \pm}^{*}}\right)\left(\frac{R T / F}{\left|z_{ \pm}\right| E_{\Omega}}\right)\left(\frac{L^{2}}{D_{ \pm}}\right) .
$$

The fractional concentration difference is expectedly of order unity (cf. Equation 15). The diffusivity of $\mathrm{HCO}_{3}{ }^{-}$ is quite similar to that of $\mathrm{Na}^{+}\left(D_{ \pm} \sim 1.2 \times 10^{-9} \mathrm{~m}^{2} / \mathrm{s}\right)$; this allows $\tau_{M}$ to be estimated through independent consideration of one supporting ion or the other. Setting $E_{\Omega} \sim 0.1 \mathrm{~V}$ to $1 \mathrm{~V}$, we obtain $\tau_{M}$ of order $10^{3}$ or $10^{4}$ seconds at most. This time scale is well below the diffusive time scale $L^{2} / D_{ \pm} \sim 10^{5} \mathrm{~s}$, but it is still comparable, if not larger, than the time scale of our experiments. We therefore conclude that the continuous rise of the cell voltage in time should be rightfully attributed, in part, to the migration-driven increase of the ohmic overpotential in time.

It is worth mentioning that the electric double layer, and all effects associated with it, have been excluded throughout this paper. This was deemed reasonable on the basis that the ionic adsorption by the electrode surface has little impact on the macroscopic distribution of the ionic species several Debye screening lengths away from the electrode. In addition, the formation of the double layer is simply too fast. Such time can be quantified by first computing the capacitance of the double layer, $C_{d}=\varepsilon / \lambda_{d} \sim 10 \mu \mathrm{F} / \mathrm{cm}^{2}$, where $\varepsilon=710$ $\mathrm{pF} / \mathrm{m}$ is the permitivity of water and $\lambda_{d}=3 \mathrm{~nm}$ is the double layer thickness. The latter is equal to one Debye screening length [31],

$$
\lambda_{d}=\frac{\varepsilon R T}{F^{2} \sum_{j} C_{j}^{*} z_{j}^{2}} .
$$

Taking $R_{\text {cell }}=E / I \sim 400 \mathrm{k} \Omega$ as the cell resistance, the cell relaxation time associated with the charging of the double electric layer is $\tau_{d}=A C_{d} R_{\text {cell }} \sim 2 \mathrm{~ms}$ [32]. The charging time is thus too short, by all accounts, to have any credible contribution to the transient nature of the cell potential over several thousand seconds. Moreover, we find that the capacitive charging current, estimated as $I_{d}=A C_{d} \mathrm{~d} E / \mathrm{d} t$ [33], is of the order of nanoamperes at best. It therefore constitutes a negligible portion of the measured current.

\section{Conclusions}

A novel method to promote gas evolution away from the electrode surface has been tested under alkaline water electrolysis. It consists in a ring microelectrode encircling a hydrophobic microcavity. The ring microelectrode does not suffer from bubble coverage, owing to the fact that bubbles preferably form on the cavity instead. Consequently, the chronopotentiometric fluctuations of the electrolysis cell have been observed to be much weaker than those associated with conventional microelectrodes. 
It has been shown that the bubbles on the cavity exert a salubrious influence on the cell potential difference by reducing the concentration overpotential of the microelectrode during the diffusive growth and detachment stages. The reduction in surface overpotential comes at the cost of a lesser gas evolution efficiency. We have argued that bubble formation also delays the concentration overpotential from reaching a steady state value. Therefore, we hold bubble formation partly accountable for the long-term transient behaviour of the cell voltage.

The second factor responsible for such long-term behaviour is the unsteadiness of the ohmic overpotential, which must conform to the large relaxation time of ionic migration across the solution in the cell. Furthermore, we have exposed the shielding effect by which bubbles increase the ohmic overpotential, which is naturally alleviated upon bubble detachment. The magnitude of the ohmic losses and the blockage effect are drastically intensified with insufficient excess of supporting electrolyte.

The insight and fundamental understanding that this contribution offers forms a useful base for future work concerning the optimal design and operation of ring microelectrodes. We believe that these findings can have important implications, for instance, in the design and advancement of gas-evolving electrochemical energy conversion systems.

\section{Acknowledgments}

The authors would like to thank S. Schlautmann for the substrate fabrication. P. v. d. L. wishes to acknowledge the assistance of F. J. Navarro Fuentes, L. Oyarte Galves, H. Witteveen, K. Cu and R. Sanders. This work was supported by the Netherlands Center for Multiscale Catalytic Energy Conversion (MCEC), an NWO Gravitation programme funded by the Ministry of Education, Culture and Science of the government of the Netherlands.

\section{Appendix A. Sample fabrication}

The fabrication method is best explained with reference to Figure A-1, which depicts the cross section of the substrate after the key steps in its fabrication process. In step 1, a silicon wafer (containing multiple samples) with a $150 \mathrm{~nm}$ thick $\mathrm{SiO}_{2}$ layer is patterned using standard photolithography techniques. The Olin OIR 907-17 resist is spin-coated, followed by DC-sputtering of a $10 \mathrm{~nm}$ thick tantalum adhesion layer and a $100 \mathrm{~nm}$ thick platinum layer with a custom-made sputter coater (T'COaty, Nanolab, MESA+ Institute). Step 2 shows the substrate after the deposited metal has been patterned via a lift-off process. The resulting metal film forms a ring shaped electrode with an inner radius of $400 \mu \mathrm{m}$ and outer radius of $410 \mu \mathrm{m}$. A superhydrophobic cavity, $30 \mu \mathrm{m}$ in diameter, is defined and created (steps 3 and 4) in the center of each electrode. The fabrication of superhydrophobic cavities has been described elsewhere [8, 19]. Finally, in step 5 the wafer is cleaned with acetone in an ultrasonic bath (VWR Ultrasonic Cleaner USC-THD, $45 \mathrm{kHz}$ ) to remove the photoresist and diced (Dicing Saw Loadpoint Micro Ace 3) to extract the $10 \times 10 \mathrm{~mm}^{2}$ samples.

\section{Appendix B. Geometric factor}

The geometric factor $\beta$, defined in Equation (12), can be verbally interpreted as the current density ratio between a disk/ring electrode and a hemispherical electrode of the same (outer) radius $r_{e}$ required to sustain a given steady-state concentration difference between the electrode surface and the bulk. It stands to reason that thinner rings will have higher values $\beta$, primarily because they posses less surface area. The geometric factor was computed numerically in FreeFem ++ by solving the steady-state diffusion equation (see Supporting Information). Values of $\beta$ for a wide range of ring thicknesses are graphed in Figure A-2. The analytical value $\beta=4 / \pi$ for a flat disk $\left(r_{i} / r_{e}=0\right)$ [28] has also been plotted. The dependance of $\beta$ on the ring thickness is well described by the fitting relation

$$
\beta=\frac{4}{\pi}\left[1+\frac{1}{3}\left(\frac{r_{i} / r_{e}}{1-r_{i} / r_{e}}\right)\right] .
$$

\section{References}

[1] W. Xu, Z. Lu, X. Sun, L. Jiang, and X. Duan. Superwetting electrodes for gas-involving electrocatalysis. Acc. Chem. Res., 51(7), 1590 (2018).

[2] S. Ardo, D. Fernandez Rivas, M. A. Modestino, et al. Pathways to electrochemical solar-hydrogen technologies. Energy Environ. Sci. (2018).

[3] J. A. Leistra and P. J. Sides. Voltage components at gas evolving electrodes. J. Electrochem. Soc., 134(10), 2442 (1987).

[4] X. Zhao, H. Ren, and L. Luo. Gas bubbles in electrochemical gas evolution reactions. Langmuir, 35(16), $5392(2019)$.

[5] A. Taqieddin, R. Nazari, L. Rajic, and A. Alshawabkeh. Physicochemical hydrodynamics of gas bubbles in two phase electrochemical systems. J. Electrochem. Soc., 164(13), E448 (2017). 
[6] C. Brussieux, P. Viers, H. Roustan, and M. Rakib. Controlled electrochemical gas bubble release from electrodes entirely and partially covered with hydrophobic materials. Electrochim. Acta, 56(20), 7194 (2011).

[7] N. Bremond, M. Arora, C.-D. Ohl, and D. Lohse. Controlled multibubble surface cavitation. Phys. Rev. Lett., 96(224501), 1 (2006).

[8] P. van der Linde, Á. Moreno Soto, P. Peñas López, J. Rodríguez-Rodríguez, D. Lohse, J. G. E. Gardeniers, D. van der Meer, and D. Fernández Rivas. Electrolysis-driven and pressure-controlled diffusive growth of successive bubbles on microstructured surfaces. Langmuir, 33(45), 12873 (2017).

[9] N. Pande, G. Mul, D. Lohse, and B. Mei. Correlating the short-time current response of a hydrogen evolving nickel electrode to bubble growth. J. Electrochem. Soc., 166(10), E280 (2019).

[10] C. Gabrielli, F. Huet, and R. P. Noqueira. Fluctuations of concentration overpotential generated at gasevolving electrodes. Electrochim. Acta, 50, 3726 (2005).

[11] X. Yang, F. Karnbach, M. Uhlemann, S. Odenbach, and K. Eckert. Dynamics of single hydrogen bubbles at a platinum microelectrode. Langmuir, 31(29), 8184 (2015).

[12] D. Fernández, P. Maurer, M. Martine, J. M. D. Coey, and M. E. Möbius. Bubble formation at a gas-evolving microelectrode. Langmuir, 30, 13065 (2014).

[13] S. D. Lubetkin. The fundamentals of bubble evolution. Chem. Soc. Rev., 24, 243 (1995).

[14] D. M. F. Santos, C. A. C. Sequeira, and J. L. Figueiredo. Hydrogen production by alkaline water electrolysis. Química Nova, 36(8), 1176 (2013).

[15] M. Schalenbach, G. Tjarks, M. Carmo, W. Lueke, M. Mueller, and D. Stolten. Acidic or alkaline? towards a new perspective on the efficiency of water electrolysis. J. Electrochem. Soc., 163(11), F3197 (2016).

[16] C. Coutanceau, S. Baranton, and T. Audichon. Chapter 3 - Hydrogen production from water electrolysis. In Hydrogen Electrochemical Production, Hydrogen Energy and Fuel Cells Primers, p. 17 - 62. Academic Press (2018).

[17] A. J. Bard and L. R. Faulkner. Electrochemical Methods: Fundamentals and Applications. John Wiley \& Sons, Inc, 2nd edition (2001).

[18] H. Vogt. The rate of gas evolution of electrodes-I. An estimate of the efficiency of gas evolution from the supersaturation of electrolyte adjacent to a gas-evolving electrode. Electrochim. Acta, 29(2), 167 (1984).

[19] P. van der Linde, P. Peñas López, Á. Moreno Soto, D. van der Meer, D. Lohse, H. Gardeniers, and D. Fernández Rivas. Gas bubble evolution on microstructured silicon substrates. Energy Environ. Sci., 11, 3452 (2018).

[20] P. S. Epstein and M. S. Plesset. On the stability of gas bubbles in liquid-gas solutions. J. Chem. Phys., 18,1505 (1950).

[21] H. F. A. Verhaart, R. M. de Jonge, and S. J. D. van Stralen. Growth rate of a gas bubble during electrolysis in supersaturated liquid. Int. J. Heat Mass Transfer, 23, 293 (1979).

[22] W. Sheng, H. A. Gasteiger, and Y. Shao-Horn. Hydrogen oxidation and evolution reaction kinetics on platinum: acid vs alkaline electrolytes. J. Electrochem. Soc., 157(11), B1529 (2010).

[23] E. J. F. Dickinson, J. G. Limon-Petersen, and R. G. Compton. The electroneutrality approximation in electrochemistry. J. Solid State Electr., 15(7-8), 1335 (2011).

[24] H. Vogt and K. Stephan. Local microprocesses at gas-evolving electrodes and their influence on mass transfer. Electrochim. Acta, 155, 348 (2015).

[25] P. T. H. M. Verhallen, L. J. P. Oomen, A. J. J. M. v.d. Elsen, J. Kruger, and J. M. H. Fortuin. The diffusion coefficients of helium, hydrogen, oxygen and nitrogen in water determined from the permeability of a stagnant liquid layer in the quasi-steady state. Chem. Eng. Sci., 39(11), 1535 (1984).

[26] F. Hecht. New development in FreeFem++. J. Numer. Math., 20(3-4), 251 (2012).

[27] J. Dukovic and C. W. Tobias. The influence of attached bubbles on potential drop and current distribution at gas-evolving electrodes. J. Electrochem. Soc., 134, 331 (1987).

[28] J. Newman. Resistance for flow of current to a disk. J. Electrochem. Soc., 113(5), 501 (1966).

[29] C. W. Tobias, M. Eisenberg, and C. R. Wilke. Diffusion and convection in electrolysis - a theoretical review. J. Electrochem. Soc., 99(12), 359C (1952).

[30] K. B. Oldham and C. G. Zoski. Mass transport to electrodes. In Comprehensive Chemical Kinetics, volume 26, p. 79-143. Elsevier (1986).

[31] K. J. Vetter. Chapter 1 - Electrochemical thermodynamics. In Electrochemical Kinetics, p. 1-103. Academic Press (1967).

[32] M. Z. Bazant, K. Thornton, and A. Ajdari. Diffuse-charge dynamics in electrochemical systems. Phys. Rev. E, 70(2), 021506 (2004).

[33] T. Berzins and P. Delahay. Kinetics of fast electrode reactions. J. Am. Chem. Soc., 77(24), 6448 (1955). 


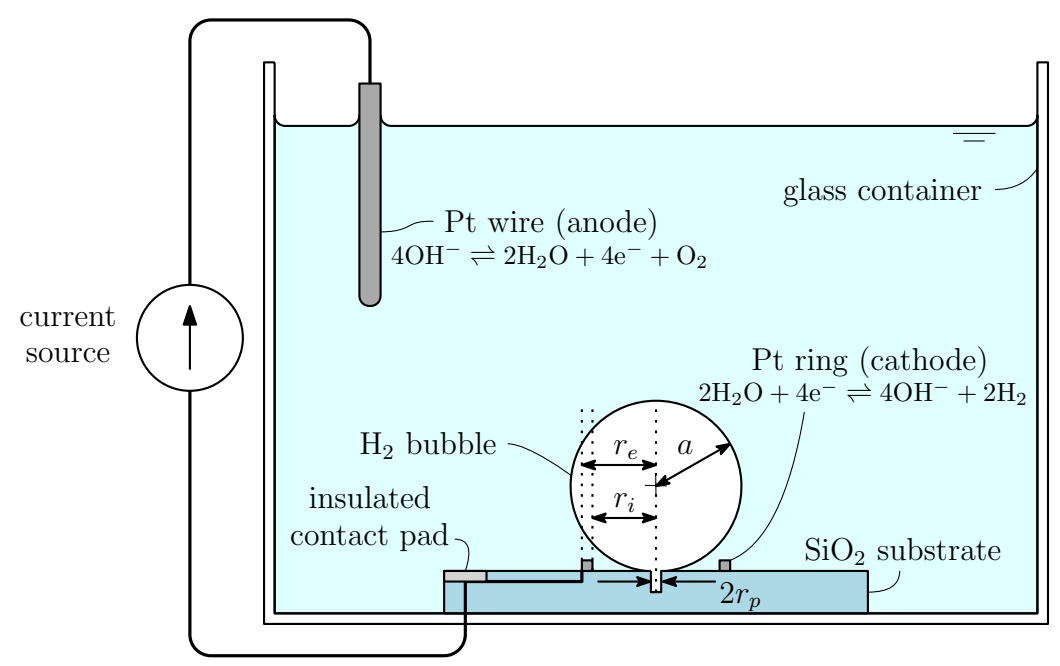

Figure 1: Sketch of the alkaline water electrolysis cell.
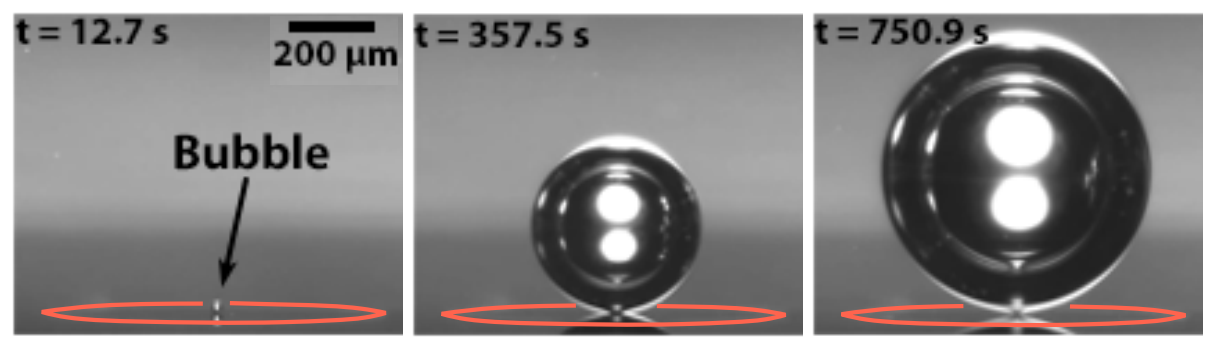

Figure 2: Sequence of images, taken at a time $t$ since the start of electrolysis, showing a hydrogen bubble nucleating and growing from the hydrophobic micropit of the $\mathrm{SiO}_{2}$ substrate. The ring electrode encircling the pit has been highlighted for clarity. The current density is $39.3 \mathrm{~mA} / \mathrm{cm}^{2}$. 


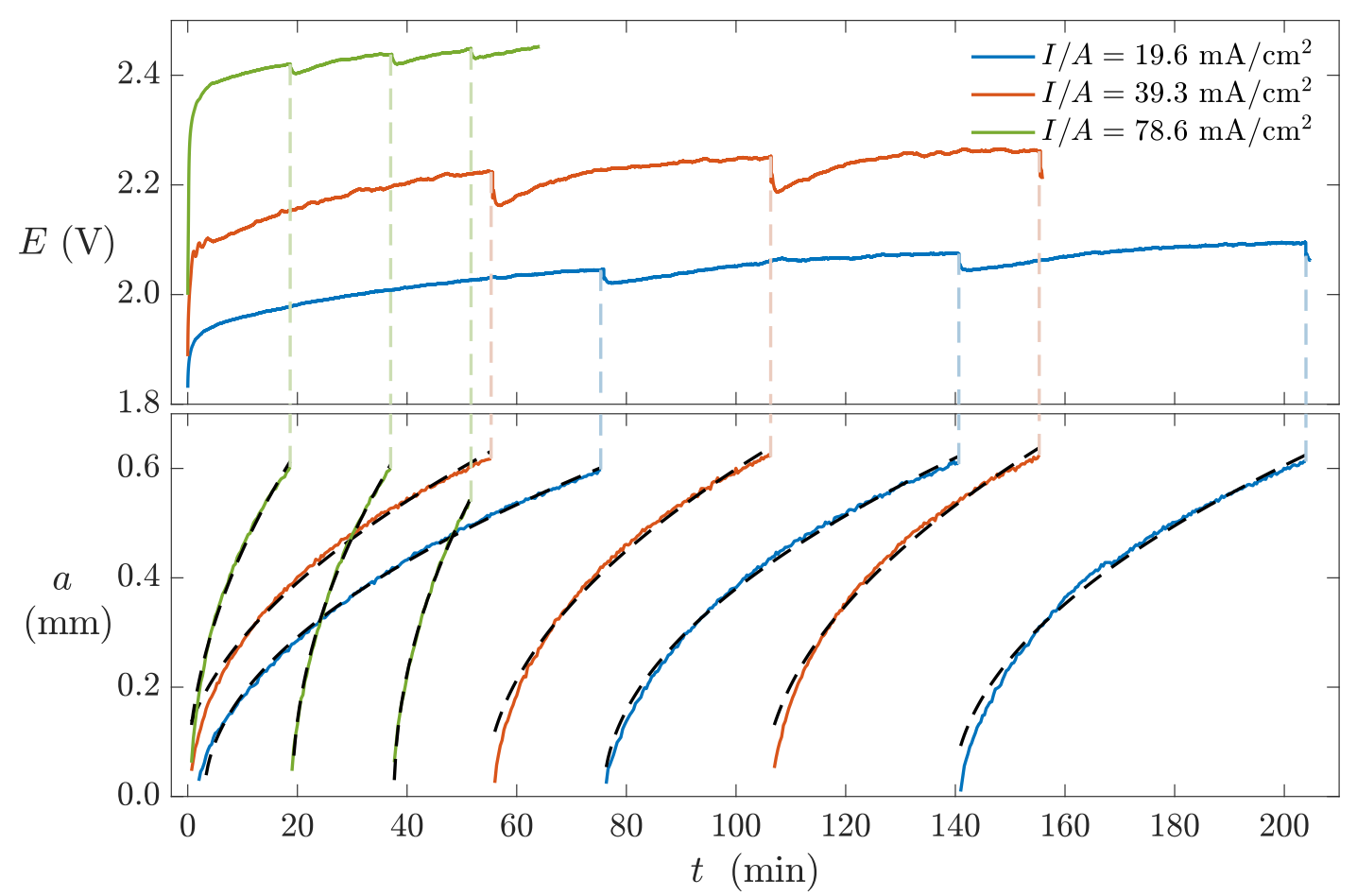

Figure 3: Cell potential $E$ and bubble radius $a$ of the first three bubbles in the succession plotted against elapsed time since the start of constant-current electrolysis. Three experiments at different current densities are shown. The dashed black curves are fits of the form $a_{n}^{2} / t_{n}=m_{n}=$ const., from which the effective supersaturation $\zeta_{n}=m_{n} / 2 \Lambda D_{\mathrm{H}_{2}}$ was then computed.

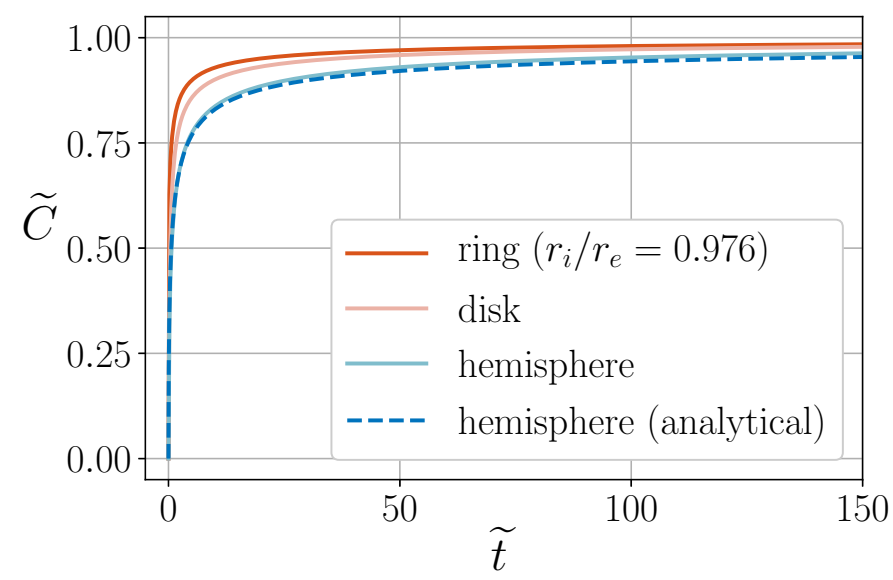

Figure 4: Numerical solution of the dimensionless surface concentration, $\widetilde{C}=\left(C_{\mathrm{H}_{2}}-C_{\mathrm{H}_{2}}^{*}\right) /\left(C_{\mathrm{H}_{2}}^{s s}-C_{\mathrm{H}_{2}}^{*}\right)$ as a function of dimensionless time $\widetilde{t}=D_{\mathrm{H}_{2}} t / r_{e}^{2}$ for the case of a thin ring, a disk and a hemispherical electrode of radius $r_{e}$. The dashed curve is the analytical solution given in (11). 

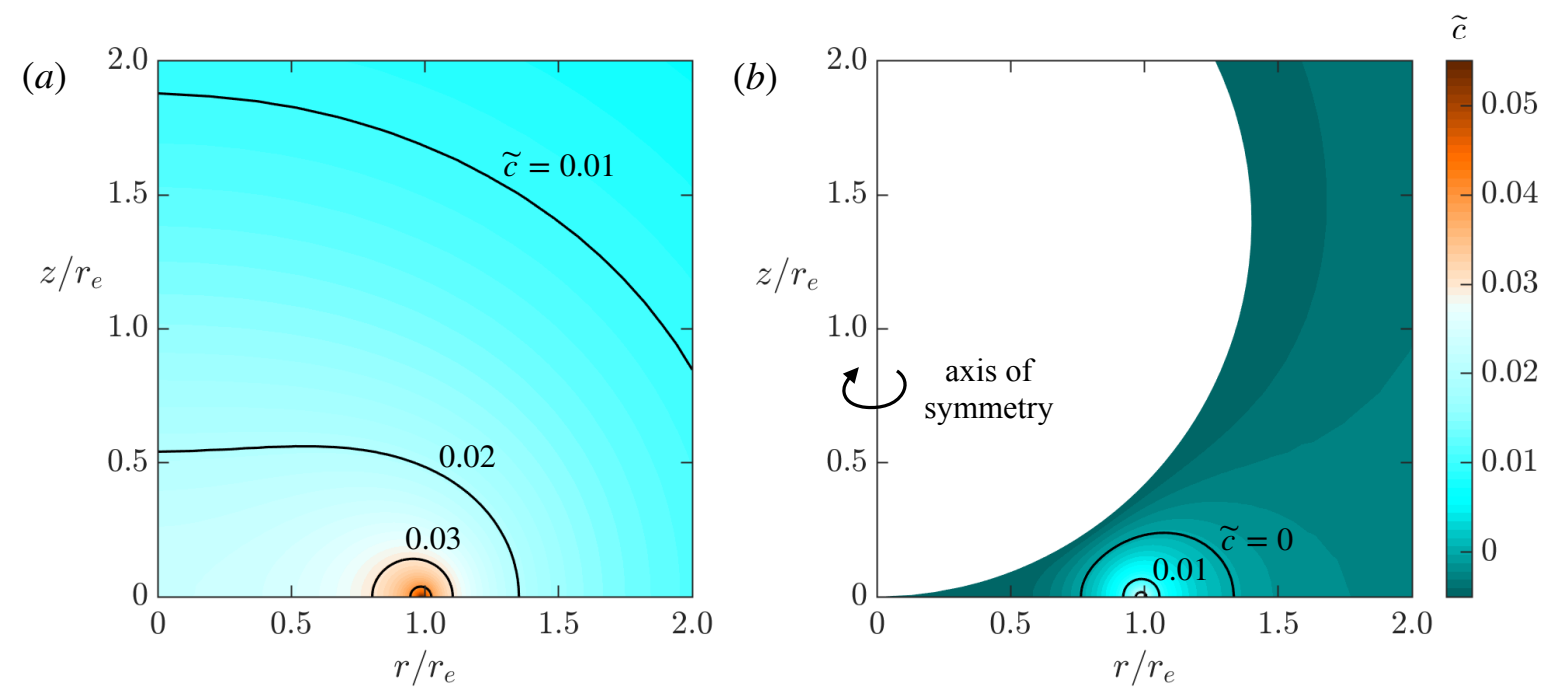

Figure 5: Snapshots at $D_{\mathrm{H}_{2}} t / r_{e}^{2}=100$ of the dimensionless concentration field of $\mathrm{H}_{2}$ gas in the vicinity of the ring electrode in $(a)$ the absence and $(b)$ the presence of an encircled bubble. The concentration has been nondimensionalised as $\widetilde{c}=n_{\mathrm{H}_{2}} F D_{\mathrm{H}_{2}} A\left(c_{\mathrm{H}_{2}}-C_{\mathrm{H}_{2}}^{*}\right) / I r_{e}$ (see Supporting Information). The initial supersaturation $(\widetilde{c}=0)$ is set at $\zeta^{*}=C_{\mathrm{H}_{2}}^{*} / k_{\mathrm{H}_{2}} p-1=0.5$, similar to the effective supersaturation observed in our experiments at the lowest current density. We take $I / A=19$ $\mathrm{mA} / \mathrm{cm}^{2}$ and the dimensionless interfacial concentration at the bubble (saturation concentration) is correspondingly set to $\widetilde{c}=-0.005$. The electrode thickness is that of our experiments, $r_{i} / r_{e}=0.976$. The $\widetilde{c}=0,0.01,0.02$ and 0.03 contours have been outlined for clarity.

(c)

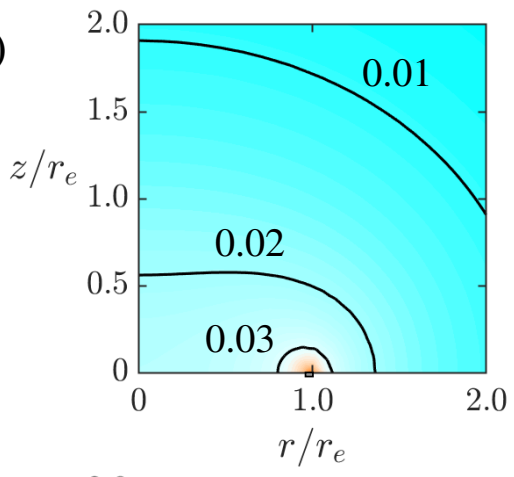

(a)

(b)

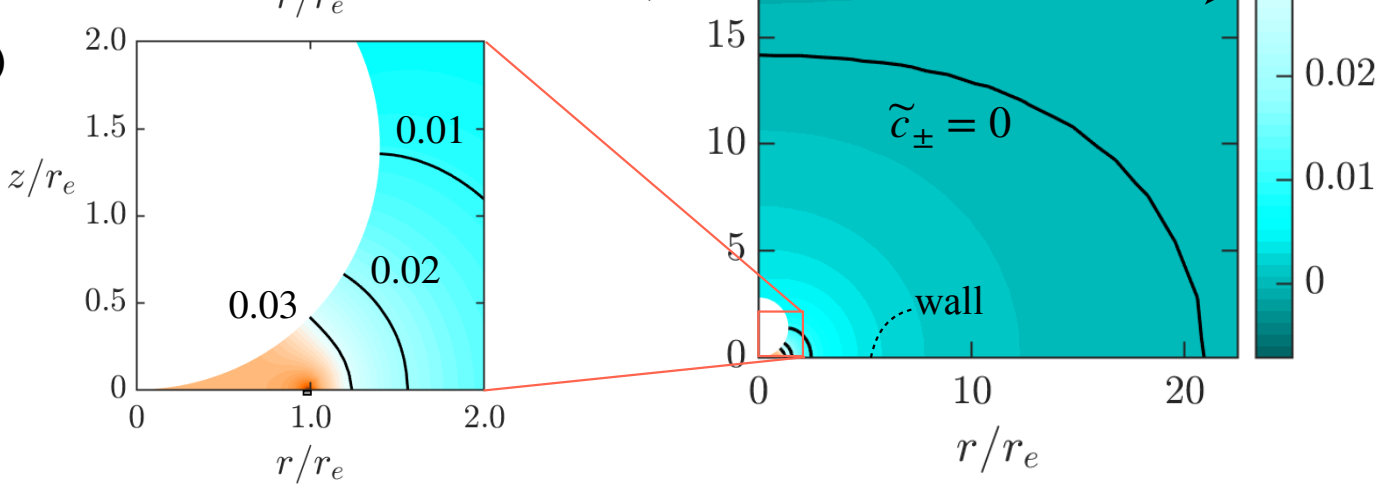

Figure 6: (a) Steady-state concentration field of cations or equivalently anions, in an axisymmetric closed domain of similar dimensions to our experimental cell. Panel $(b)$ shows a close-up of the ionic concentration field in the vicinity of the ring electrode, whereas panel $(c)$ shows the same close-up in the absence of the bubble. The concentration has been nondimensionalised as $\widetilde{c}=2 F D_{-} A\left(c_{ \pm}-\right.$ $\left.C_{ \pm}^{*}\right) / I r_{e}$ (see Supporting Information). The initial bulk concentration corresponds to $\widetilde{c}=0$; the 0 , $0.01,0.02$ and 0.03 contours have been outlined for clarity. 

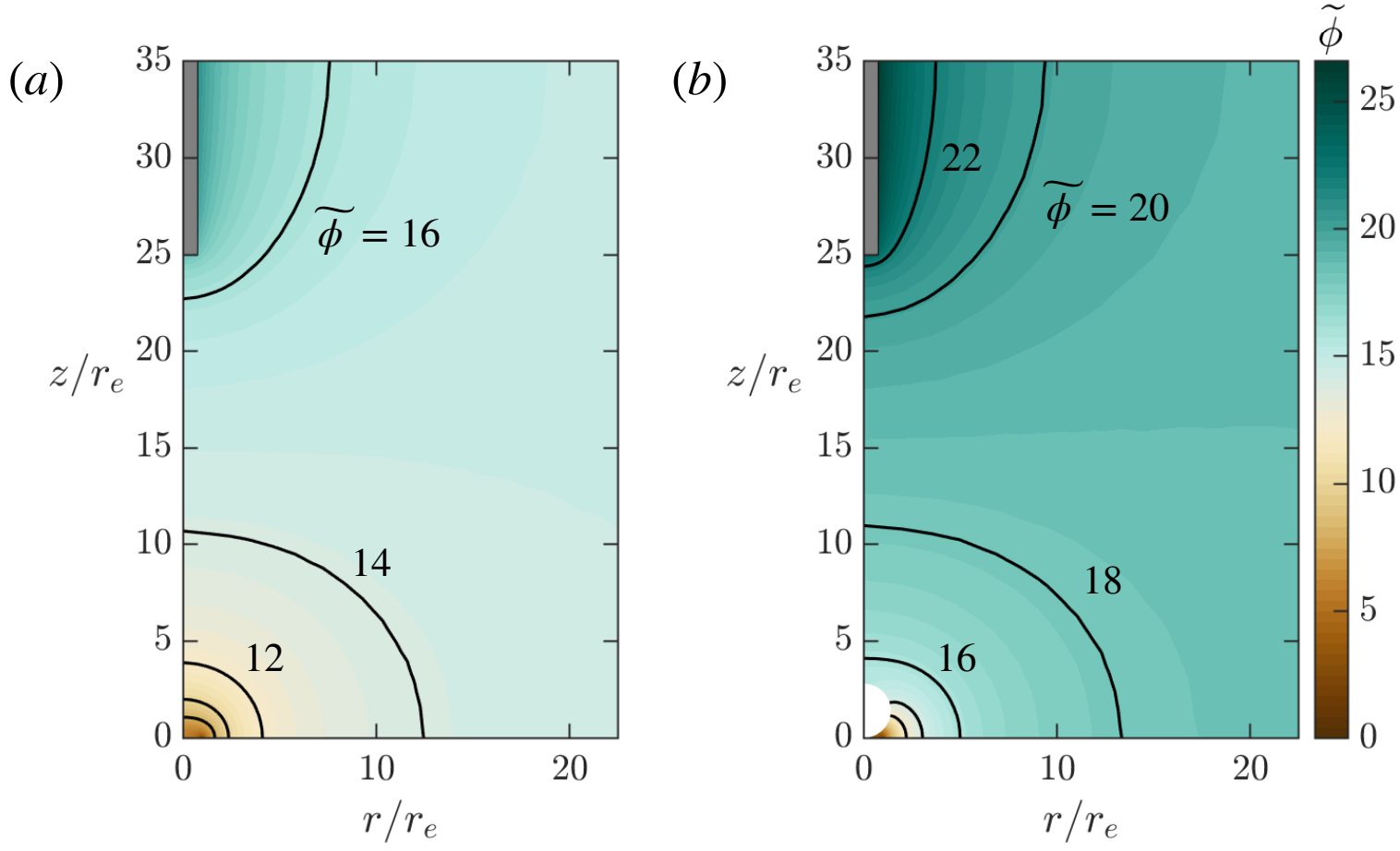

Figure 7: Steady-state electric potential field in $(a)$ the absence and in $(b)$ the presence of a bubble for electrolyte concentration level $\Gamma=0.002$. The electric potential has been nondimensionalised as $\widetilde{\phi}=F \phi / R T$, and shifted in order to make the potential zero at the cathode. The contours for $\widetilde{\phi}=8$, $10, \ldots 22$ have been outlined for clarity. The location of the cathode and anode are as indicated in Figure 6 .

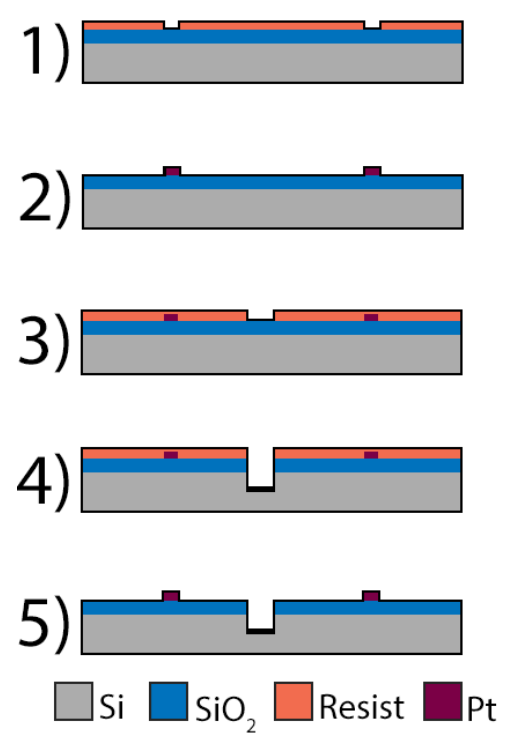

Figure A-1: Cross-sectional overview of the fabrication steps (not to scale). 


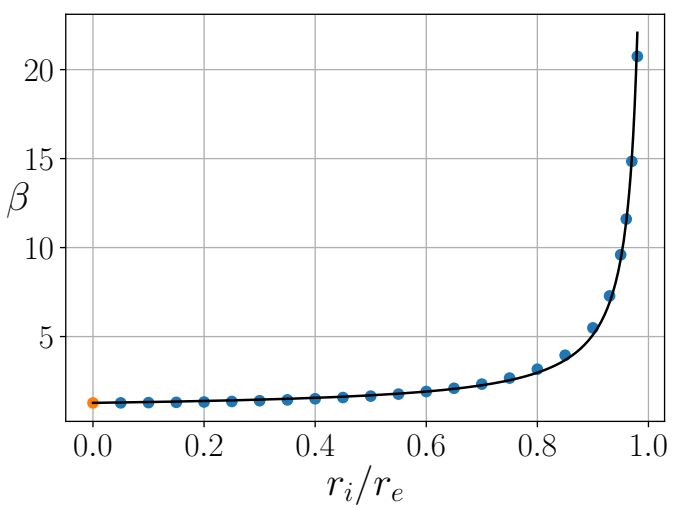

Figure A-2: Numerical values of $\beta$ (dots) as a function of the ring thickness. The fitting curve is given by Equation (B-2). 


\section{Supporting Information for "Decoupling Gas Evolution from Water-Splitting Electrodes"}

Pablo Peñas ${ }^{\mathrm{a}}$, Peter van der Linde ${ }^{\mathrm{b}}$, Wouter Vijselaar ${ }^{\mathrm{d}}$, Devaraj van der Meer ${ }^{\mathrm{a}}$, Detlef Lohse ${ }^{\mathrm{a}}$, Jurriaan Huskens $^{\mathrm{d}}$, Han Gardeniers ${ }^{\mathrm{b}}$, Miguel A. Modestino ${ }^{\mathrm{c}}$, and David Fernández Rivas ${ }^{\mathrm{b}}$

${ }^{a}$ Physics of Fluids Group and Max Planck Center Twente, MESA+ Institute and J. M. Burgers Centre for Fluid Dynamics, Faculty of Science and Technology, University of Twente, P.O. Box 217, 7500 AE Enschede, The Netherlands

${ }^{\mathrm{b}}$ Mesoscale Chemical Systems, MESA + Institute, University of Twente, Enschede, The Netherlands

${ }^{\mathrm{c}}$ New York University, Tandon School of Engineering, 6 MetroTech Center, Brooklyn, NY 11201, United States of America

${ }^{\mathrm{d}}$ Molecular Nanofabrication, University of Twente, Enschede, The Netherlands

\section{Transport of dissolved hydrogen gas}

In this first section we present the mathematical formulation implemented in the main manuscript to numerically compute (i) the time-evolving $\mathrm{H}_{2}$ concentration field in the presence of a bubble and (ii) the ring geometric factor $\beta$ by means of the steady-state concentration field.

\section{Concentration field in the presence of a bubble}

The concentration of dissolved $\mathrm{H}_{2}$ gas evolves according to the diffusion equation,

$$
\frac{\partial c_{\mathrm{H}_{2}}}{\partial t}=D_{\mathrm{H}_{2}} \nabla^{2} c_{\mathrm{H}_{2}}
$$

subject to the initial condition $c_{\mathrm{H}_{2}}(\boldsymbol{x}, t=0)=C_{\mathrm{H}_{2}}^{*}$ and boundary conditions

$$
\begin{array}{cc}
c=k_{\mathrm{H}_{2}} p, & \text { on the bubble surface, } \\
\frac{\partial c_{\mathrm{H}_{2}}}{\partial x}=-\frac{I / A}{n_{\mathrm{H}_{2}} F D_{\mathrm{H}_{2}}}, & \text { on the cathode surface, } \\
\frac{\partial c_{\mathrm{H}_{2}}}{\partial x}=0, & \text { on the remaning boundaries. }
\end{array}
$$

Here $x$ denotes the coordinate pointing normally outwards (into the fluid) from the surface. Note that $C_{\mathrm{H}_{2}}^{*}$ is some initial bulk concentration, assumed uniform, and that the remaining boundaries consist of the substrate surface, axis of symmetry and infinity. On the bubble surface, which is treated as a stationary sphere by virtue of the pseudosteady approximation (cf. main manuscript), the dissolved concentration of $\mathrm{H}_{2}$ gas corresponds to the saturation concentration of $\mathrm{H}_{2}$ in water at a given pressure $p$. Henry's coefficient of $\mathrm{H}_{2}$ in water is $k_{\mathrm{H}_{2}} \simeq 7.7 \times 10^{-6}$ $\mathrm{mol} / \mathrm{m}^{3} \mathrm{~Pa}$ at room temperature.

Let us now define a dimensionless concentration and time as

$$
\widetilde{c}=\frac{n_{\mathrm{H}_{2}} F D_{\mathrm{H}_{2}}}{(I / A) r_{e}}\left(c_{\mathrm{H}_{2}}-C_{\mathrm{H}_{2}}^{*}\right), \quad \widetilde{t}=\frac{D_{\mathrm{H}_{2}} t}{r_{e}^{2}} .
$$

Additionally, let $\widetilde{\boldsymbol{\nabla}}=r_{e} \boldsymbol{\nabla}$ and $\widetilde{x}=x / r_{e}$. The system in dimensionless form becomes

$$
\frac{\partial \widetilde{c}}{\partial \widetilde{t}}=\widetilde{\nabla}^{2} \widetilde{c}
$$

subject to the initial condition $\widetilde{c}(\widetilde{\boldsymbol{x}}, \widetilde{t}=0)=0$ and boundary conditions

$$
\begin{array}{rlrl}
\widetilde{c}=\frac{n_{\mathrm{H}_{2}} F D_{\mathrm{H}_{2}}}{(I / A) r_{e}}\left(k_{\mathrm{H}_{2}} p-C_{\mathrm{H}_{2}}^{*}\right), & & \text { on the bubble surface, } \\
\frac{\partial \widetilde{c}}{\partial \widetilde{x}} & =-1, & & \text { on the cathode surface, } \\
\frac{\partial \widetilde{c}}{\partial \widetilde{x}} & =0, & & \text { on the remaning boundaries. }
\end{array}
$$




\section{Geometric factor}

The geometric factor was computed numerically from the steady-state diffusion equation

$$
\nabla^{2} c_{\mathrm{H}_{2}}=0
$$

on a large (ideally semi-infinite) axisymmetric domain with boundary conditions

$$
\begin{array}{ll}
c_{\mathrm{H}_{2}}=C_{\mathrm{H}_{2}}^{s s}, & \text { on the (ring) cathode surface, } \\
\frac{\partial c_{\mathrm{H}_{2}}}{\partial x}=0, & \text { on the substrate and axis of symmetry, } \\
c_{\mathrm{H}_{2}}=C_{\mathrm{H}_{2}}^{*}, & \text { at infinity. }
\end{array}
$$

The steady-state concentration on the electrode surface is denoted by $C_{\mathrm{H}_{2}}^{s s}$ whereas $C_{\mathrm{H}_{2}}^{*}$ is the initial bulk concentration, assumed uniform. It is convenient to work with the following dimensionless concentration,

$$
\widetilde{c}=\frac{c_{\mathrm{H}_{2}}-C_{\mathrm{H}_{2}}^{*}}{C_{\mathrm{H}_{2}}^{s s}-C_{\mathrm{H}_{2}}^{*}}
$$

together with $\widetilde{\nabla}=r_{e} \boldsymbol{\nabla}$ and $\widetilde{x}=x / r_{e}$. The dimensionless system becomes $\widetilde{\nabla}^{2} \widetilde{c}=0$, with $\widetilde{c}=1$ on the electrode surface, $\widetilde{c}=0$ at inifinity and zero flux at the substrate surface and axis of symmetry. Such a system was solved in a large axisymmetric $100 r_{e}$ square domain. The geometric factor $\beta$ is mathematically defined as the mean concentration flux (the flux is usually not uniform) across the electrode surface. For a horizontal ring of outer radius $r_{e}$ and inner radius $r_{i}$, the factor can be numerically computed as

$$
\beta=-\left.\frac{1}{\pi\left(1-r_{i}^{2} / r_{e}^{2}\right)} \int_{r_{i} / r_{e}}^{1} 2 \pi \frac{\partial \widetilde{c}}{\partial \widetilde{z}}\right|_{\widetilde{z}=0} \mathrm{~d} \widetilde{r}
$$

where $(\widetilde{r}, \widetilde{z})=\left(r / r_{e}, z / r_{e}\right)$ are dimensionless cylindrical coordinates.

\section{A note on the boundary conditions employed on the electrode surface}

The simulations reported in the main sections of the manuscript assume that the current density at the electrodes is uniform. In other words, we impose a uniform concentration or potential gradient (Neumann-type) boundary condition at the electrodes, rather than the more realistic (Dirichlet-type) boundary condition of uniform concentration or potential that we use for instance to obtain $\beta$. The reason is simplicity: galvanostatic conditions imply that the value of the mean current density is known. Hence, the values of the mean surface concentration and potential fluxes are also known; the values of the surface concentration and potential are not. It is imperative to realise that both conditions are strictly incompatible with one another (except if the domain is 1D or spherically symmetric), meaning for example that the current density on the ring electrode cannot be uniform indeed if the potential is known to be uniform everywhere on its surface. Consequently, one should be aware that the solution changes slightly when switching from one boundary condition to the other.

\section{Steady-state ionic transport model}

In this second section we will derive the equations that are employed in the main manuscript to compute the steady-state ionic concentration and electric potential fields within our constant-current water electrolysis cell. The layout of the axisymmetric numerical domain is sketched in Figure S1(a). It will be shown that under a specific set of assumptions and approximations, the steady-state transport equations and boundary conditions simplify to those found in Figure S1(b).

\section{Governing equations}

The ionic transport within the electrolyte solution is governed by the Nernst-Planck equation,

$$
\frac{\partial c_{j}}{\partial t}=D_{j} \nabla^{2} c_{j}+\nabla \cdot\left(\frac{D_{j} z_{j} F}{R T} c_{j} \nabla \phi\right)-\boldsymbol{U} \cdot \boldsymbol{\nabla} c_{j}+S_{j}
$$

where $c_{j}$ is the molar concentration and $D_{j}$ is the diffusivity of species $j=\mathrm{Na}^{+}, \mathrm{HCO}_{3}{ }^{-}, \mathrm{CO}_{3}{ }^{2-}, \mathrm{OH}^{-}$and $\mathrm{H}_{3} \mathrm{O}^{+}$, these being the main ions present in a sodium bicarbonate solution. The electric potential is denoted by $\phi$ whereas $z_{j}$ is the charge of the species, $R=8.314 \mathrm{Jmol}^{-1} \mathrm{~K}^{-1}$ is the gas constant, $T=293 \mathrm{~K}$ the absolute temperature and $F=96485 \mathrm{C} / \mathrm{mol}$ is Faraday's constant. Electroneutrality implies

$$
\sum_{j} z_{j} c_{j}=0
$$


$(a)$

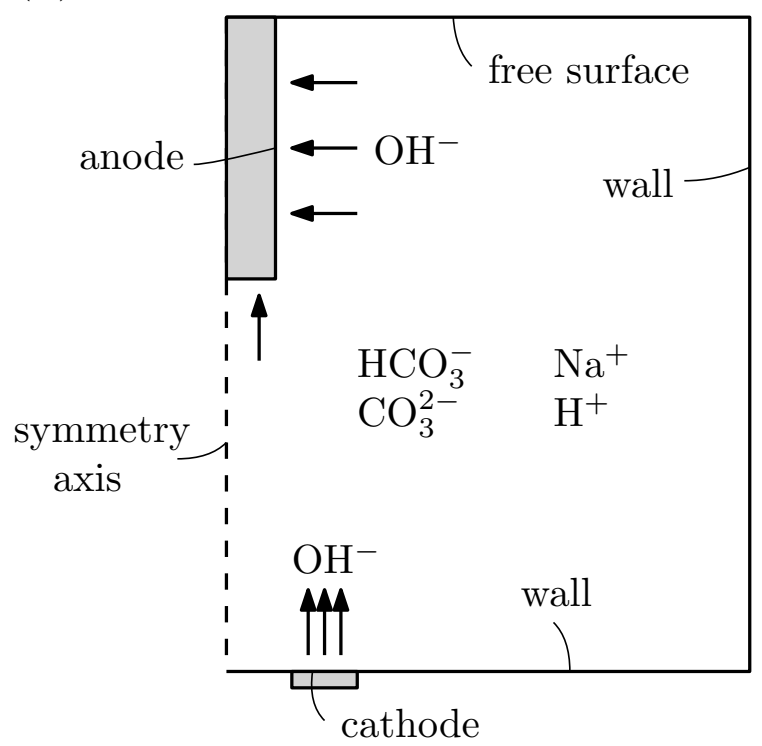

(b)

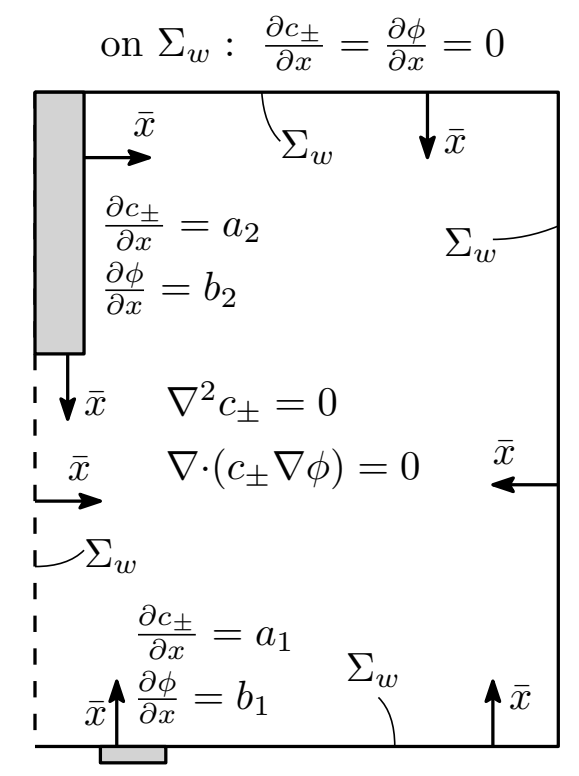

Figure S1: (a) Sketch of axisymmetric cell undergoing alkaline water electrolysis, in which $\mathrm{OH}^{-}$ ions are produced and consumed at the cathode and anode, respectively. Other ions present in sodium bicarbonate electrolyte solution are also indicated. (b) Steady-state transport equations and boundary conditions of the total concentration of cations or anions, $c_{ \pm}$, and the electric potential field $\phi$. Symbols $a_{1}, b_{1}, a_{2}$, and $b_{2}$ are constants given by equations (S31)-(S34). Vector $\bar{x}$ denotes the unit normal pointing out from the cell boundaries.

The flow velocity field, if any, is denoted by $\boldsymbol{U}$. The equilibrium reactions between the different ions are taken into account through the source term $S_{j}$. In this context, $S_{j}$ is the local production or recombination rate per unit volume of species $j$. Charge is conserved throughout all equilibrium reactions. Therefore,

$$
\sum_{j} z_{j} S_{j}=0
$$

In sodium bicarbonate solution, the following chemical equilibrium reactions are known to take place:

$$
\begin{array}{rc}
\mathrm{H}_{2} \mathrm{CO}_{3}+\mathrm{OH}^{-} \rightleftharpoons \mathrm{HCO}_{3}{ }^{-}+\mathrm{H}_{2} \mathrm{O}, & \mathrm{pK}_{\mathrm{b} 1}=7.65 \\
\mathrm{HCO}_{3}{ }^{-}+\mathrm{OH}^{-} \rightleftharpoons \mathrm{CO}_{3}{ }^{2-}+\mathrm{H}_{2} \mathrm{O}, & \mathrm{pK}_{\mathrm{b} 2}=3.67 \\
\mathrm{H}_{3} \mathrm{O}^{+}+\mathrm{OH}^{-} \rightleftharpoons 2 \mathrm{H}_{2} \mathrm{O}, & \mathrm{pK}_{\mathrm{w}}=14
\end{array}
$$

Most of the carbonic acid breaks down into molecules of dissolved $\mathrm{CO}_{2}$ and water: $\mathrm{H}_{2} \mathrm{CO}_{3} \rightarrow \mathrm{CO}_{2}+\mathrm{H}_{2} \mathrm{O}$. The base dissociation constant is related to the concentrations through

$$
\mathrm{K}_{\mathrm{b}}=\frac{\left[\mathrm{HB}^{+}\right]\left[\mathrm{OH}^{-}\right]}{[\mathrm{B}]}
$$

for an acid-base reaction of the form: $\mathrm{HB}^{+}+\mathrm{OH}^{-} \rightleftharpoons \mathrm{B}+\mathrm{H}_{2} \mathrm{O}$. Additionally, $\mathrm{K}_{\mathrm{w}}=\left[\mathrm{H}_{3} \mathrm{O}^{+}\right]\left[\mathrm{OH}^{-}\right]$.

\section{Equilibrium limits: infinite and zero buffer capacity}

In a $0.010 \mathrm{M}$ sodium bicarbonate solution of $\mathrm{pH} 7.4$, the equilibrium reactions (S19)-(S21) yield the following equilibrium concentrations:

$$
C_{\mathrm{Na}^{+}}^{*}=C_{\mathrm{HCO}_{3}{ }^{-}}^{*}=0.010 \mathrm{M}, \quad C_{\mathrm{CO}_{3}{ }^{2-}}^{*}=1.2 \times 10^{-5} \mathrm{M}, \quad C_{\mathrm{OH}^{-}}^{*}=2.5 \times 10^{-7} \mathrm{M}, \quad C_{\mathrm{H}_{3} \mathrm{O}^{+}}^{*}=4.0 \times 10^{-8} \mathrm{M} .
$$

In the alkaline electrolysis cell, $\mathrm{OH}^{-}$ions are produced at the cathode and consumed at the anode. We therefore expect an equilibrium overconcentration of $\mathrm{OH}^{-}$ions (with respect to $C_{\mathrm{OH}^{-}}^{*}$ ) in the vicinity of the cathode, and an underconcentration in the vicinity of the cathode. An excess of hydroxyl ions favours their recombination into carbonate and bicarbonate ions, and viceversa, in compliance with the equilibrium reactions. The equilibrium concentrations of $\mathrm{HCO}_{3}{ }^{-}, \mathrm{CO}_{3}{ }^{2-}$ and $\mathrm{OH}^{-}$will readjust to oppose a given change in $\mathrm{OH}^{-}$concentration, $\Delta C_{\mathrm{OH}^{-}}$, as shown in Figure S2.

It is seen that when $\Delta C_{\mathrm{OH}^{-}}<10^{-3} \mathrm{M}$, the concentration of the predominant species $\left(\mathrm{HCO}_{3}{ }^{-}\right)$stays largely invariant. Moreover, the increment of the $\mathrm{OH}^{-}$equilibrium concentration remains very small. In this regime, the 

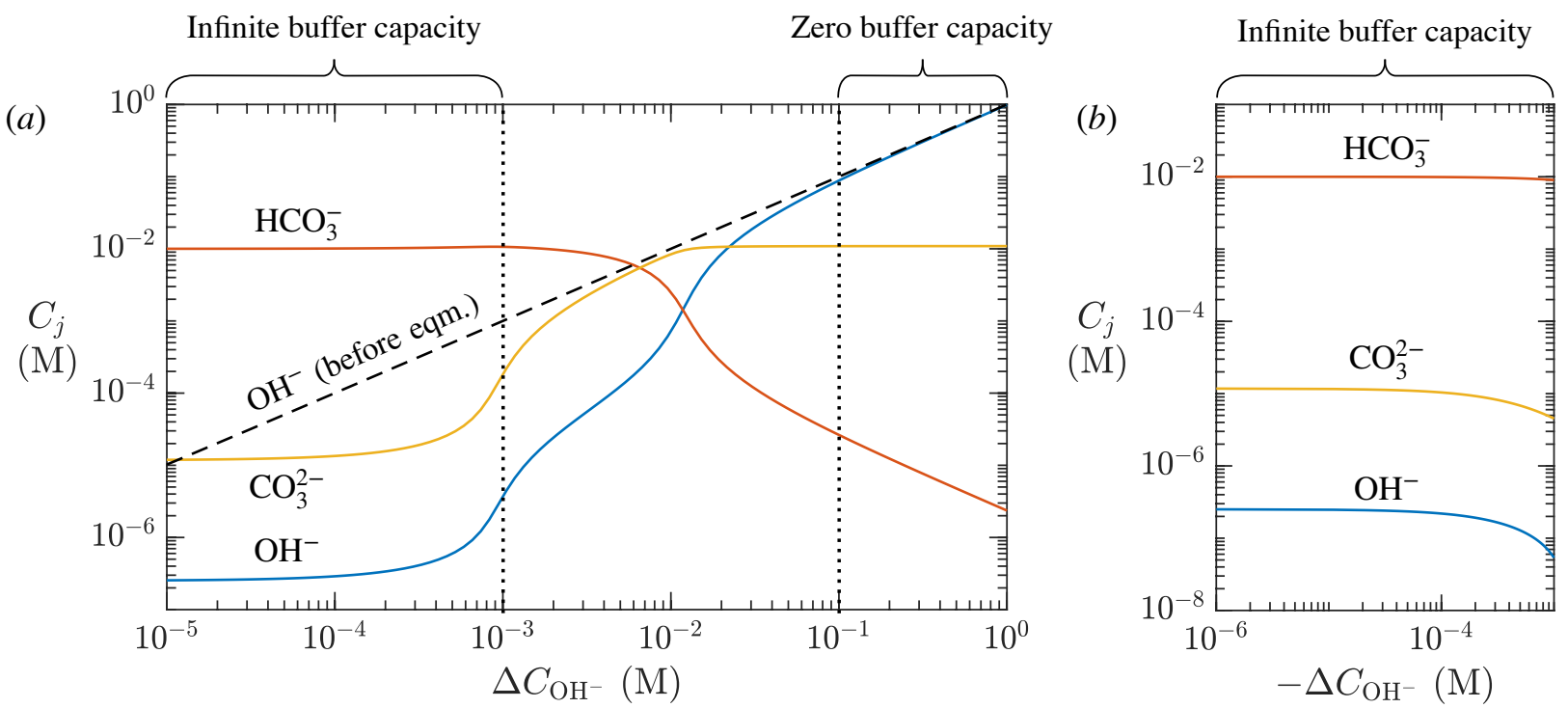

Figure S2: Equilibrium concentrations of species $j=\mathrm{HCO}_{3}{ }^{-}, \mathrm{CO}_{3}{ }^{2-}$ and $\mathrm{OH}^{-}$that result from a sudden (local) concentration change $\Delta C_{\mathrm{OH}^{-}}$from the initial equilibrium value $C_{\mathrm{OH}^{-}}^{*} \cdot(a)$ Response to a sudden overconcentration, $\Delta C_{\mathrm{OH}^{-}}>0$ (cathode) and (b) a sudden underconcentration $\Delta C_{\mathrm{OH}^{-}}<0$ (anode). The dashed line indicates the sudden concentration of $\mathrm{OH}^{-}$, i.e., in the absence of equilibrium reactions.

solution can be treated as a perfect buffer. It stands to reason that the source term in the transport equation for $\mathrm{HCO}_{3}{ }^{-}$must be much smaller than the diffusion or migration terms.

Hereon let us denote the sum of some property over all cations and all anions with subscripts + and respectively, i.e., for a given quantity $X$,

$$
X_{-}=X_{\mathrm{HCO}_{3}-}+X_{\mathrm{OH}^{-}}+X_{\mathrm{CO}_{3}{ }^{2-}}, \quad X_{+}=X_{\mathrm{Na}^{+}}+X_{\mathrm{H}_{3} \mathrm{O}^{+}}
$$

Since the hydronium concentration is very low, the cation concentration is $c_{+} \simeq c_{\mathrm{Na}^{+}}$. In the limit of infinite buffer capacity, where $\Delta C_{\mathrm{OH}^{-}}<10^{-3} \mathrm{M}$, we have that $\mathrm{HCO}_{3}{ }^{-}$is the predominant anion. Hence,

$$
c_{-} \simeq c_{\mathrm{HCO}_{3}-}, \quad(S / D)_{-}=0, \quad c_{-}=c_{+}
$$

Note that the third equality is established by electroneutrality, assuming that the solution essentially contains only homovalent ions (in this case $\left|z_{+}\right|=\left|z_{-}\right|=1$ ). As $\Delta C_{\mathrm{OH}^{-}}$increases beyond $10^{-3} \mathrm{M}$, the solution gradually losses its buffer capacity. When $\Delta C_{\mathrm{OH}^{-}}>10^{-1} \mathrm{M}$, the buffer solution has lost all its buffering capacity and $\mathrm{OH}^{-}$ becomes the predominant species. The source term in the transport equation for $\mathrm{OH}^{-}$is therefore negligible. In the limit of zero buffer capacity,

$$
c_{-} \simeq c_{\mathrm{OH}^{-}}, \quad(S / D)_{-}=0, \quad c_{-}=c_{+}
$$

\section{Derivation of the model equations}

In the steady state and in the absence of advection, the sum of the Nernst-Planck equation (S16) for all charged species gives

$$
\nabla^{2}\left(c_{+}+c_{-}\right)=(S / D)_{+}+(S / D)_{-}
$$

Note that the migration terms have cancelled out by virtue of electroneutrality. Since $c_{+} \simeq c_{\mathrm{Na}^{+}}$, the cations do not undergo any equilibrium reaction, hence $(S / D)_{+}=0$. We will assume that the electrolyte behaves as a perfect buffer. This is justified since the small current densities employed ensure that almost everywhere in the solution the variation in ionic concentration remains small, namely $\Delta C_{-}^{*} / C_{-}^{*} \ll 1$. It should be pointed out that this assumption breaks down first within the thin diffusion layer closest to the ring cathode surface where the local concentrations of $\mathrm{OH}^{-}$and $\mathrm{CO}_{3}{ }^{2-}$ are highest, even possibly exceeding the concentration of $\mathrm{HCO}_{3}{ }^{-}$if the current density is large enough. Under the limit of infinite buffer capacity, equation (S27) simplifies to

$$
\nabla^{2} c_{ \pm}=0
$$

where $c_{ \pm}=c_{+}=c_{-}$denotes the total concentration of cations or anions. To satisfy the Nernst-Planck equation in (S16), it follows from Equation (S28) that

$$
\boldsymbol{\nabla} \cdot\left(c_{ \pm} \boldsymbol{\nabla} \phi\right)=0
$$


from which the electric potential field $\phi$ may be calculated.

The boundary conditions for $c_{ \pm}$and $\phi$ at the cathode are prescribed under the assumptions of infinite buffer capacity and uniform current density. The former assumption implies that the relevant diffusion coefficient $D_{-}$ that determines the ionic boundary layer thickness at the electrodes is that of $\mathrm{HCO}_{3}{ }^{-}$as opposed to that of $\mathrm{OH}^{-}$. The latter assumption implies the imposition of constant flux: $J_{\mathrm{OH}^{-}}=I / A F n_{\mathrm{OH}^{-}}$. One has that

$$
\begin{aligned}
\frac{I / A}{F D_{-} n_{\mathrm{OH}^{-}}} & =-\frac{\partial c_{-}}{\partial x}-\frac{z_{-} F}{R T} C_{-} \frac{\partial \phi}{\partial x}, \\
0 & =-\frac{\partial c_{+}}{\partial x}-\frac{z_{+} F}{R T} C_{+} \frac{\partial \phi}{\partial x},
\end{aligned}
$$

where $x$ is the coordinate in the normal direction pointing outwards from the cathode surface, whereas $C_{-}$and $C_{+}$denote the ionic concentrations evaluated on the cathode surface. Noting that $C_{-}=C_{+}=C_{ \pm}, z_{+}=-z_{-}=1$ and $n_{\mathrm{OH}^{-}}=1$, the two conditions in Equation (S30) can be added to obtain a single boundary condition for $c_{ \pm}$,

$$
\frac{\partial c_{ \pm}}{\partial x}=-\frac{I / A}{2 F D_{\mathrm{HCO}_{3}-}} .
$$

Substitution of Equation (S31) into Equation (S30) finally yields the boundary condition for the electric potential,

$$
\frac{F}{R T} \frac{\partial \phi}{\partial x}=\frac{I / A}{2 F D_{-} C_{ \pm}},
$$

Similarly, at the anode we have

$$
\frac{\partial c_{ \pm}}{\partial x}=\frac{I / A_{a}}{2 F D_{-}}
$$

and

$$
\frac{F}{R T} \frac{\partial \phi}{\partial x}=-\frac{I / A_{a}}{2 F D_{-} C_{ \pm}},
$$

where $A_{a}$ is the anode area and $C_{ \pm}$is now the concentration on the anode surface. Zero concentration flux and zero potential flux boundary conditions apply on the remaning boundaries (including the bubble surface), cf. Figure $\mathrm{S} 1(b)$. The total number of ionic species, equal to $C_{ \pm}^{*} V$ (with $C_{ \pm}^{*}$ the initial bulk concentration of cations or anions and $V$ the solution volume), is thereby conserved.

\section{Nondimensionalisation of the model equations}

Let us define the dimensionless counterparts of the ionic concentration, bulk concentration, and electric potential as follows:

$$
\widetilde{c}_{ \pm}=\frac{2 F D_{-}}{(I / A) r_{e}}\left(c_{ \pm}-C_{ \pm}^{*}\right), \quad \widetilde{C}_{ \pm}^{*}=\frac{2 F D_{-} C_{ \pm}^{*}}{(I / A) r_{e}}, \quad \widetilde{\phi}=F \phi / R T .
$$

In dimensionless form, the governing model equations become

$$
\begin{array}{r}
\widetilde{\nabla}^{2} \widetilde{c}_{ \pm}=0, \\
\widetilde{\nabla} \cdot\left[\left(\widetilde{c}_{ \pm}+\widetilde{C}_{ \pm}^{*}\right) \widetilde{\nabla} \widetilde{\phi}\right]=0,
\end{array}
$$

where $\widetilde{\boldsymbol{\nabla}}=r_{e} \boldsymbol{\nabla}$ and $\widetilde{x}=x / r_{e}$. Boundary conditions on the cathode surface are

$$
\frac{\partial \widetilde{c}_{ \pm}}{\partial \widetilde{x}}=-1, \quad \frac{\partial \widetilde{\phi}}{\partial \widetilde{x}}=\frac{1}{\widetilde{C}_{ \pm}+\widetilde{C}_{ \pm}^{*}},
$$

where $\widetilde{C}_{ \pm}$is the dimensionless concentration at the cathode surface. On the anode surface we have

$$
\frac{\partial \widetilde{c}_{ \pm}}{\partial \widetilde{x}}=A / A_{a}, \quad \frac{\partial \widetilde{\phi}}{\partial \widetilde{x}}=-\frac{A / A_{a}}{\widetilde{C}_{ \pm}+\widetilde{C}_{ \pm}^{*}}
$$

where $\widetilde{C}_{ \pm}$now denotes the dimensionless concentration at the anode surface. The electrolyte concentration level $\Gamma$ defined in Equation (16) of the main manuscript is related to $C_{ \pm}^{*}$ through $\Gamma=C_{ \pm}^{*} r_{e} / L$. In our case a concentration level of $\Gamma=0.002$ is therefore equivalent to setting $C_{ \pm}^{*}=0.05$ since $L / r_{e}=25$. 
